\title{
Skin Cancer Diagnostic using Machine Learning Techniques - Shearlet Transform and Naïve Bayes Classifier
}

\author{
S. Mohan Kumar, J. Ram Kumar, K. Gopalakrishnan
}

\begin{abstract}
Development of abnormal cells in the skin is known as skin cancer or melanoma, which can spread other parts of the body. Melanoma rarely occurs in eye, mouth and intestines. In this study, the classification of melanoma using shearlet transform coefficients and naïve Bayes classifier is discussed. The melanoma images are decomposed by the shearlet transform. Then, from the shearlet coefficients, predefined number of (50, 75 and 100) coefficients are selected from the decomposed subbands. The selected subband coefficients are directly applied to the naïve Bayes classifier. Performance of skin cancer classification system is measured in terms of accuracy. Results show that a better classification accuracy of $\mathbf{9 0 . 5} \%$ is achieved at 3rd level with 100 coefficients of shearlet transform and naïve Bayes classifier for skin image classification system.
\end{abstract}

Keywords: Melanoma, Shearlet transform, Subband coefficients, Naïve Bayes classifier.

\section{INTRODUCTION}

The abnormal growth of cell which can't control by healthy cells by a transformation in the skin is known as skin cancer. The early diagnosis of skin cancer is required because it spreads fast. Convolution Neural Network (CNN) based Skin lesion classification with novel regularizer is discussed in [1]. Initially, the skin lesion images are fed into CNN for training. Novel regularizer method is used for classification. The evaluation of skin lesion change is discussed in [2] by accurate segmentation and registration techniques. Diameter features, border, shape and asymmetry features are extracted. Scale-invariant feature transform is used for segmentation.

Skin dermoscopic image classification using multiple CNN is discussed in [3]. The softmax layer is used for classification. Skin cancer classification system using deep learning is described in [4]. The alexnet layer in the CNN perfoms the convolution in each layer and classification is made by softmax layer.

Revised Manuscript Received on December 30, 2019.

* Correspondence Author

Dr. S. Mohan Kumar, Department of Mechanical Engineering, Indian Institute of Technology, Kanpur, India. Email:drsmohankumar@gmail.com

Dr. J. Ram Kumar, Department of Mechanical Engineering, Indian Institute of Technology, Kanpur,India.E-Mail: jrkumar@iitk.ac.in

Dr. K. Gopalakrishnan, New Horizon College of Engineering, Bangalore, Karnataka,India.E-Mail:profgoki@gmail.com

(C) The Authors. Published by Blue Eyes Intelligence Engineering and Sciences Publication (BEIESP). This is an open access article under the CC BY-NC-ND license (http://creativecommons.org/licenses/by-nc-nd/4.0/)
Skin lesion classification using deep ensemble learning is described in [5]. Neural network architecture is used for feature extraction. ResNet-50 and Inception V3 architecture are used for classification. Effective diagnosis of melanoma

skin cancer using dermoscopy images is described in [6]. Asymmetry, color, border irregularity and diameter features are used. Classification is made by backpropagation neural network.

Skin disease classification using deep CNN and multi-class Support Vector Machine (SVM) is described in [7]. The feature extraction step is made by deep CNN and error-correcting output codes. The machine learning SVM classifier is used for classification. Skin lesion analysis using CNN is described in [8]. The deep CNN architecture with color histogram distribution, multiscale color local binary pattern and edge histogram is used to extract features. The random forest classifier is used for classification.

Image classification based on deep learning for medical diagnosis is described in [9]. CNN extracts the features with alexnet and googlenet. Supervised learning classifier is used for classification. Skin cancer detection using various classifiers is described in [10]. The input skin images are preprocessed by histogram adjustment, contrast improvement, grayscale conversion and noise filtering. Thresholding technique is used for segmentation. The extracted features are geometric circularity features, texture features, wavelet transform. SVM, k-nearest neighbor classifier are used.

Decision support system for skin cancer classification is described in [11]. The asymmetry, border, color and diameter rule is applied for feature extraction. Then k-nearest neighbor, Bayesian and multilayer perceptron is used for classification. Combination of three different features is used for skin cancer classification is discussed in [12]. Shape features, color features and histogram of oriented gradients and fractal features are combined to extract the features. SVM classifier is used for classification.

In this work, an efficient method for skin cancer classification system using dermoscopic images by shearlet transform coefficients and naïve Bayes classifier is presented. The organization of this paper as follows: In section 2, materials and methods for skin cancer classification system are given. The result and discussion of skin image classification in terms of accuracy is explained in section 3. The last section concludes the skin cancer classification system. 


\section{PROPOSED METHOD}

The workflow of skin cancer classification system is shown in figure 1 . In this technique, shearlet coefficient selection using rank features and classification using naïve Bayes classifier are employed. The input skin cancer images are given to the transformation module, which decomposes the given input images to obtain the sub-band coefficients. After that feature extraction stage selects the features according to their rank by t-test. Then the selected features are classified by naïve Bayes classifier as normal or abnormal.

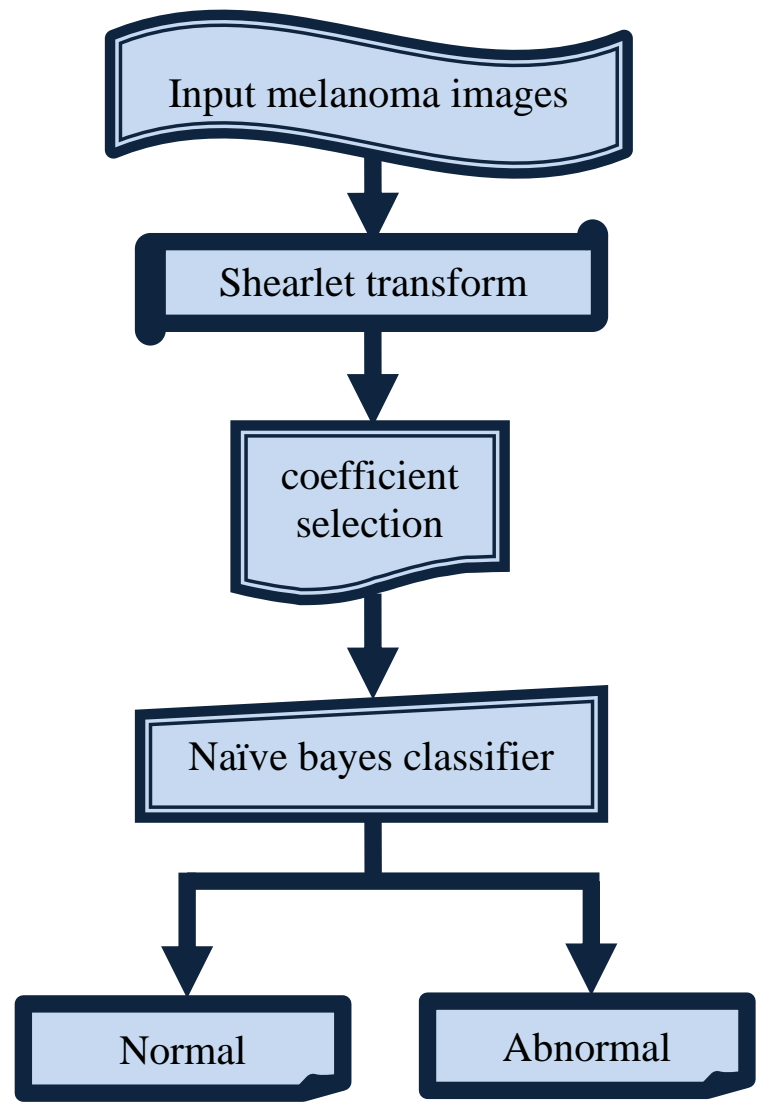

Fig. 1.Work flow of the skin cancer image classification system.

\section{A. Shearlet Decomposition}

Good Shearlets are a multiscale framework. It is constructed by parabolic scaling, translation and shearing and applied to some generating functions. In multivariate problem, shearlets allows to encode the efficient anisotropic features. Shearlets used for sparse approximation functions $f \in S^{2}\left(K^{2}\right)$. It is a natural wavelet extension that contains the multivariate functions. The shearlet transform is obtained by,

$$
\left\|f-f_{E}\right\|_{S^{2}}^{2} \leq L E^{-2}(\log E)^{3}, \quad E \rightarrow \infty,
$$

where the constant $L$ denotes the maximum curvature of the curve for the magnitudes of $f, f^{\prime}$ and $f^{\prime \prime}$.The shearlet transform is used in other fields like mammogram classification [13], Face recognition technique [14] and satellite image denoising [15]. In this study, shearlet transform is used to decompose the skin cancer image

features.

\section{B. Coefficient Selection from Shearlet Transform}

All The coefficients are selected from the shearlet decomposed sub-bands by applying statistical t-test and sort them as maximum to minimum in sub-band individually. From a large number of shearlet coefficients, the first 50, 75 and 100 coefficients are selected. These selected coefficients are given as inputs for classification.

\section{Naïve Bayes Classification}

Naïve Bayes classifier is a simple machine learning classifier algorithm. It makes the classification posterior in Bayesian algorithm. It is represented by the Bayesian network. It is used as a solution for spam and text detection. Naïve Bayes classifier can perform more complicated classification methods. The Bayes rule is,

$$
P(K / L)=\frac{P(L / K) P(K)}{P(L)}
$$

Naïve Bayes classifier performs well for large number of data points because finding the parameters for probability functions can be done quickly. Naïve Bayes classifier is used in other fields like eligibility of blood donor's and emotion recognition [16-17] and heart disease prediction system [18]. It is used to classify the selected coefficients.

\section{RESULTS AND DISCUSSION}

The performance is evaluated by using the $\mathrm{PH} 2$ database, which is freely downloadable from the PH2 database link [19]. It contains 100 dermoscopic color images with melanocytic lesions. The resolution of dermoscopic images is $768 \times 560$ pixels. The sample normal and abnormal images in PH2 database is shown in figure 2.
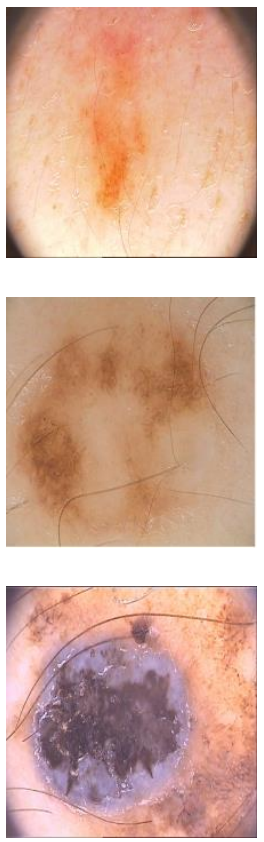

Fig. 2.Sample normal and abnormal images in $\mathrm{PH} 2$ database

(a) Normal images, (b) Benign images and (c) Malignant images.

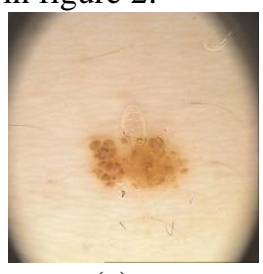

(a)

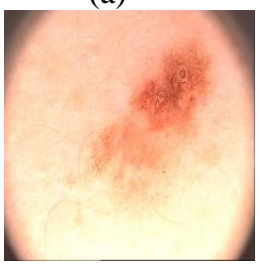

(b)
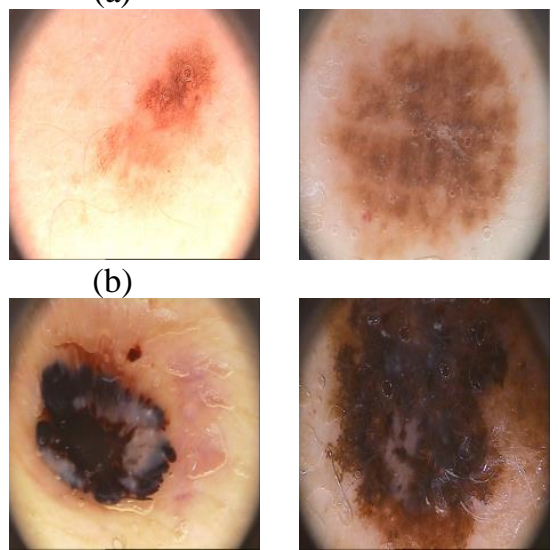

\& Sciences Publication 
The shearlet transform is applied to extract the features of input skin cancer images, and they are decomposed into different sub-band. The first 50, 75 and 100 coefficients are selected from shearlet sub-bands and used for classification.
Also, performance is evaluated in terms of accuracy. Table 1 shows the accuracies obtained by using 50, 75 and 100 coefficients of 4 th levels of shearlet transform.

Table- I: Classification accuracies obtained by shearlet coefficients using naïve Bayes classifier

\begin{tabular}{|c|c|c|c|}
\hline \multirow{2}{*}{$\begin{array}{c}\text { Shearlet transform } \\
\text { Decomposition level }\end{array}$} & \multicolumn{3}{|c|}{ Naïve Bayes Classification accuracy (\%) } \\
\cline { 2 - 4 } & $\mathbf{5 0}$ - Coefficients & $\mathbf{7 5}$ - Coefficients & $\mathbf{1 0 0}$ - Coefficients \\
\hline 1 & 77.5 & 80.5 & 88 \\
\hline 2 & 90.5 & 87 & 95 \\
\hline 3 & 91.5 & 80.5 & 90 \\
\hline 4 & 88 & 84.5 & 90.5 \\
\hline
\end{tabular}

From Table-I, it is observed that 100 coefficients produce the highest accuracy of $95 \%$ at the 2nd level of shearlet decomposition. Also, it is observed that other 50 and 75 coefficients produce $91.5 \%$ and $84.5 \%$ of accuracy. The performance of shearlet transform with naïve Bayes classifier is shown in Fig 3.

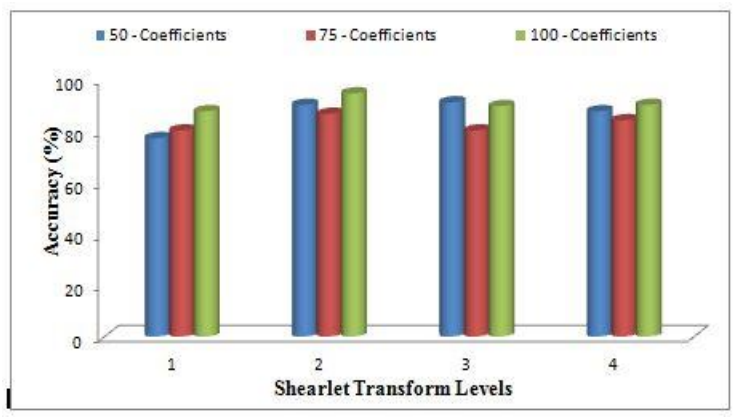

Fig. 3.Performance of shearlet transform with naïve Bayes classifier.

\section{CONCLUSION}

An efficient method for skin cancer image classification is presented. The coefficients of sub-bands are selected by rank features using the t-test. 50-coefficients, 75-coefficients and 100- coefficients are selected from different levels of shearlet decomposition. These features are given as inputs for naïve Bayes classification. Results show that the 100-coefficients produce a better classification accuracy of $95 \%$ at the 2nd level of shearlet decomposition comparing with 50 and 75 coefficients at the same level of decomposition.

\section{REFERENCES}

1. M. A. Albahar, "Skin Lesion Classification Using Convolutional Neural Network With Novel Regularizer" IEEE Access, Mar.2019, pp.38306-38313.

2. F. Navarro, M. Escudero-Vinolo , J. Bescos, "Accurate segmentation and registration of skin lesion images to evaluate lesion change" IEEE journal of biomedical and health informatics, 23(2), Apr.2018, pp.501-508.

3. Y. Guo, A. S. Ashour, L. Si, D. P. Mandalaywala, " Multiple Convolutional Neural Network for Skin Dermoscopic Image Classification", IEEE International Symposium on Signal Processing and Information Technology,Dec.2018, pp. 365-369.

4. K. M. Hosny, M. A. Kassem, M. M. Foaud, "Skin Cancer Classification using Deep Learning and Transfer Learning” ,IEEE Cairo International Biomedical Engineering Conference,Dec.2018, pp. 90-93.

5. A. H. Shahin, A. Kamal, M. A. Elattar, "Deep Ensemble Learning for Skin Lesion Classification from Dermoscopic Images” ,IEEE Cairo International Biomedical Engineering Conference, Dec.2018, pp. 150-153.

6. S. Majumder,M. A. Ullah, "Feature extraction from dermoscopy images for an effective diagnosis of melanoma skin cancer", IEEE International
Conference on Electrical and Computer Engineering, Dec.2018, pp. 185-188.

7. N. Hameed, A. M. Shabut, M. A. Hossain, "Multi-Class Skin Diseases Classification Using Deep Convolutional Neural Network and Support Vector Machine", IEEE International Conference on Software, Knowledge, Information Management \& Applications, Dec.2018, pp. $1-7$.

8. V. Singh, I. Nwogu, "Analyzing Skin Lesions in Dermoscopy Images Using Convolutional Neural Networks", IEEE International Conference on Systems, Man, and Cybernetics (SMC), Oct.2018, pp.4035-4040.

9. J. Shihadeh, A. Ansari, T. Ozunfunmi, "Deep Learning Based Image Classification for Remote Medical Diagnosi”, IEEE Global Humanitarian Technology Conference, Oct.2018, pp. 1-8.

10. P. Shahi, S. yadav, N. Singh, N. P. Singh, "Melanoma Skin Cancer Detection Using Various Classifiers", Uttar Pradesh Section International Conference on Electrical, Electronics and Computer Engineering, Nov.2018, pp. 1-5.

11. D. Ruiz, V. Berenquer, A.Soriano, B. A. SaNchez, "Decision support system for the diagnosis of melanoma:A comparative approach", Expert Systems with Applications, 38(12), Nov.2011, pp. 15217-23.

12. M. Nasir, M. Attique Khan, M. Sharif, I. U. Lali IU, T. Saba, T. Iqbal, “ An improved strategy for skin lesion detection and classification using uniform segmentation and feature selection based approach", Microscopy research and technique, 81(6), Jun.2018, pp.528-543.

13. A. M. Ibrahim, B. Baharudin, B, "Classification of mammogram images using shearlet transform and kernel principal component analysis", International Conference on Computer and Information Sciences (ICCOINS) Aug.2016, pp. 340-344.

14. C. R.Singh, H. Y. Patil, "A shearlet transform based illumination invariant 2-D face recognition" ,International Conference on Electrical, Electronics, and Optimization Techniques (ICEEOT), Mar.2016, pp 3407-3412.

15. T. S. Anju, N. N. Raj, "Satellite image denoising using shearlet transform", International Conference on Communication and Signal Processing (ICCSP),Apr.2016, pp. 0571-0575.

16. S. K. Bhakre, A. Bang, "Emotion recognition on the basis of audio signal using naive bayes classifier", International Conference on Advances in Computing, Communications and Informatics (ICACCI),Sep.2016, pp. 2363-2367.

17. W. B. Zulfikar, Y. A. Gerhana, A. F. Rahmania, "An Approach to Classify Eligibility Blood Donors Using Decision Tree and Naive Bayes Classifier", International Conference on Cyber and IT Service Management (CITSM), Aug.2018, pp. 1-5.

18. M. A. Jabbar, S. Samreen, "Heart disease prediction system based on hidden naïve bayes classifier", International Conference on Circuits, Controls, Communications and Computing (I4C), Oct.2016, pp. 1-5.

19. PH2 Database Link: https://www.fc.up.pt/addi/ph2\%20database.html

\section{AUTHORS PROFILE}

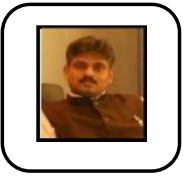

Dr. S. Mohan Kumar, M.E[CSE]., PhD[CSE] has two decades of experience in the fields of academic administration, research, Quality Assurance and also in educational consultancy. He has six years of experience as Head of the department IT/CSE. He is Editor-in-Chief of International Scientific Journal of Contemporary Research in Engineering, Science and Management. 